\title{
Estrés Traumático Secundario (ETS) en Periodistas Mexicanos y Defensores de Derechos Humanos
}

\section{Secondary Traumatic Stress (STS) in Mexican Journalists and Human Rights Defenders}

\author{
Rogelio Flores Morales* \\ Universidad Nacional Autónoma de México \\ Liliana Souza Colín \\ Universidad Autónoma Metropolitana, México \\ Ángela Oviedo Mireles \\ Jorge Fernando Bonilla Allende \\ Universidad Nacional Autónoma de México \\ (Rec.: abril de 2016 - Acept.: junio de 2016)
}

\begin{abstract}
Resumen
El objetivo del presente estudio descriptivo fue identificar la prevalencia de síntomas de estrés traumático secundario (ETS) en una muestra conjunta de periodistas mexicanos y defensores de derechos humanos $(\mathrm{N}=88)$, cuyo trabajo profesional demanda regularmente un contacto cercano con víctimas de violencia. Se encontró que 36.4\% de los participantes presentaron sintomatología "alta" o "severa" de ETS. Sin embargo, no se ubicaron diferencias significativas entre ambos grupos. Por otra parte, las mujeres y quienes laboraban más de 40 horas a la semana, sí mostraron síntomas significativamente más altos. Los resultados de esta investigación transversal reflejan el considerable desgaste psicológico que pueden generar las exposiciones secundarias en profesionistas que documentan y establecen vínculos sistemáticos con personas traumatizadas por la violencia social en México.
\end{abstract}

Palabras clave: Estrés traumático secundario; periodistas; defensores de derechos humanos; violencia; México.

\begin{abstract}
The main goal of this descriptive study was to identify the prevalence of secondary traumatic stress (STS) symptoms in a pooled sample of Mexican journalists and human right defenders $(\mathrm{N}=88)$, whose activities regularly demand a close contact with victims of violence. It was found that $36.4 \%$ of the participants presented "high" or "severe" STS symptoms. However, no significant differences between these groups of professionals were observed. Conversely, women and those who worked more than 40 hours a week presented significantly more severe symptoms. The results of this transversal investigation reflect the psychologic wear that these secondary exposures can generate in professionals who establish systematic links with subjects who have been traumatized by the social violence prevalent in modern Mexican society.
\end{abstract}

Keywords: Secondary traumatic stress; journalists; human rights defenders; violence; Mexico.

\footnotetext{
* Correspondencia a: Rogelio Flores Morales. Facultad de Psicología de la Universidad Nacional Autónoma de México (UNAM). Av. Universidad 3004, edificio "B", cubículo 218, tercer piso. Col. Copilco-Universidad. C. P. 04510. Ciudad de México, México. Tel. (52) 55-56222256. E-mail: rogeflores@yahoo.com o rogelio.flores@comunidad.unam.mx.
} 


\section{Introducción}

Los efectos psicológicos de la exposición directa o primaria a eventos traumáticos (particularmente la victimización criminal, los desastres naturales, las guerras y los actos terroristas) han sido bien documentados en diversas publicaciones científicas (Briere \& Scott, 2006; Herman, 1992; Horowitz, 1993; van der Kolk, McFarlane \& Weisaeth, 2007; Wilson \& Raphael, 1993). Sin embargo, son relativamente pocos los estudios que colocan su atención en aquellas personas traumatizadas de manera secundaria o vicaria; es decir, en individuos que han establecido un contacto cercano y sistemático con sobrevivientes o víctimas de violencia, y que a partir de esa experiencia empática desarrollaron un conjunto de malestares emocionales (Figley, 1995, 1999; Pearlman \& Saakvitne, 1995).

En efecto, la empatía es un valor del que florecen sentimientos nobles como la solidaridad y el amor. Proporciona la capacidad de mirar desde la perspectiva del otro y entender el sufrimiento (Fernádez-Pinto, López-Pérez \& Márquez, 2008). No obstante, de esta noble manifestación se puede derivar cansancio y desgaste emocional que -en casos extremos- deviene en la formación de trastornos (Bride, Robinson, Yegidis \& Figley, 2003; Figley, 1999; Moreno, Morante, Garrosa \& Rodríguez, 2004a). En el campo de la psicología se utiliza una metáfora para ilustrar este fenómeno: la existencia de una especie de "contagio emocional" (Hatfield, Cacciopo \& Rapson, 1994; Smither, 1977) que impacta en quien escucha y acompaña a las víctimas o sobrevivientes.

El estrés traumático secundario (ETS) -también conocido como desgaste por empatía- es justamente un síndrome relacionado con la exposición indirecta a algún trauma o a diversos factores secundarios de estrés (Figley, 1999; Moreno et al., 2004a). Esta entidad nosológica se caracteriza por la aparición de respuestas psicoemocionales perturbadoras que se experimentan tras haber establecido un contacto sistemático con el dolor y/o sufrimiento de otras personas. Es decir, las exposiciones indirectas a eventos traumáticos pueden producir efectos perturbadores (cognitivos, físicos y conductuales) en quienes ayudan, asisten, cuidan o establecen algún tipo de contacto sistemático o cercano con víctimas (Figley, 1999).

Charles R. Figley (1999) define al ETS como el conjunto de emociones y comportamientos naturales que surgen tras conocer en detalle un evento traumático experimentado por alguien significativo. El ETS, sin embargo, es un proceso que deviene de manera paulatina; es decir, no aparece como respuesta inmediata al primer contacto con la persona o con su historia de dolor. Es, más bien, el efecto acumulado del contacto sistemático con víctimas de violencia, sobrevivientes de catástrofes humanas o naturales, o con personas que experimentan una situación emocional muy difícil o perturbadora.

Los síntomas de ETS son casi idénticos a los del estrés postraumático (PTSD), ya que en su seno se incluyen también respuestas intrusivas, evitativas y de activación (Bride et al., 2003). No obstante, la diferencia entre ambos cuadros se ubica en la etiología, es decir, en los factores que desencadenan los síntomas. De acuerdo con Figley (1999), los efectos negativos de la exposición secundaria a un evento traumático son similares a los desencadenados por una exposición primaria, con la única diferencia de que la expresión posterior de la experiencia traumática en una persona (PTSD) se convierte (a manera de "contagio") en un evento traumático para una segunda o tercera (ETS).

Los síntomas de ETS se pueden manifestar de tres maneras diferentes: 1) Síntomas intrusivos (mediante la irrupción de recuerdos recurrentes del evento traumático, flashbacks sobre los sucesos perturbadores y/o pesadillas); 2) Síntomas evitativos (aislamiento social, anestesia emocional, desinterés en las actividades cotidianas, amnesia); y 3) Síntomas de activación (insomnio, sobresaltos, dificultad para concentrarse, irritabilidad) (Bride et al., 2003).

Se sabe que existe un costo emocional por apoyar a personas traumatizadas, y éste puede manifestarse en un deterioro de las funciones laborales, sociales y familiares de los individuos (sobre todo si no se toman medidas preventivas de autoprotección). Debido a ello, un elevado número de estudios se ha centrado, justamente, en el área preventiva (Lerias \& Byrne, 2003). Uno de los factores que contribuyen a la aparición de ETS es la carga excesiva de trabajo, lo que provoca dificultades en las relaciones sociales de quienes lo padecen (Hensel, Ruiz, Finney \& Dewa, 2015). Asimismo, se ha demostrado que haber sufrido una experiencia similar a la vivida por la persona asistida o acompañada puede propiciar la aparición de ETS en el especialista (Dunkley \& Whelan, 2006; Gardell \& Harris, 2003; Lerias \& Byrne, 2003).

Entre los tópicos examinados, se ubican estudios sobre intervenciones clínicas con fines terapéuticos (Betta, Morales, Rodriguez \& Guerra, 2007; Guerra, Fuenzalida \& Hernández, 2009; Moreno, Morante, Rodríguez \& Rodríguez, 2008), validación de instrumentos para su diagnóstico (Meda, Moreno, Rodríguez, Arias \& Palomera, 2011), revisiones generales del tema (Moreno, Morante, Garrosa \& Rodríguez, 2004b) e investigaciones sobre prevalencias en poblaciones específicas. Con respecto a estas últimas, Montero (2014) identificó una prevalencia de ETS de $36.50 \%$ en una muestra conformada por personal médico $(\mathrm{N}=82)$. Por otro lado, en un estudio de Guerra y Pereda (2015) con 259 psicólogos clínicos se encontró una prevalencia de $37.80 \%$. Por su parte, Beck y Gable (2012), aplican- 
do la Escala de Estrés Traumático Secundario (EETS) de Bride en una muestra de 464 enfermeras, hallaron que un $35 \%$ de las participantes presentaba sintomatología.

Entre las poblaciones más estudiadas se encuentran aquellos profesionales y trabajadores que cotidianamente establecen una relación cercana con víctimas de violencia o con sobrevivientes de eventos traumáticos tales como médicos, enfermeras, educadores, bomberos, paramédicos, trabajadores sociales, terapeutas de centros de ayuda infantil, abogados, profesionales de servicios de emergencia, policías y psicólogos clínicos (Adam, Boscarino \& Figley, 2006; Brady, Guy, Poelstra \& Brokaw, 1999; Courtois, 1988; Chrestman, 1999; Cunningham, 2003; Figley, 1995; Follette, Polusny \& Milbeck, 1994; Herman, 1992; Joinson, 1992; McCann \& Pearlman, 1990; Meda, Moreno, Palomera, Arias \& Vargas, 2012; Meyers \& Cornille, 2002; Solomon, Waysman, Belkin, Levy, Mikulincer \& Enoch, 1992). Sin embargo, los estudios de corte cuantitativo sobre la presencia de ETS en periodistas y/o defensores(as) de derechos humanos es prácticamente nulo (Flores-Morales \& Reidl, 2014).

Cabe destacar que a diferencia del burnout -el cual es, en efecto, un estado de cansancio físico, emocional y mental causado por un prolongado involucramiento en situaciones emocionalmente demandantes (Pines \& Aronson, 1988)- el ETS surge necesariamente de la exposición indirecta a experiencias traumáticas (Adams, Figley \& Boscarino, 2008). En este sentido, ambos constructos son cercanos pero se diferencian justamente por el carácter etiológico del desgaste (traumático vs no traumático) (Rossi et al., 2012).

Por todo lo anterior, consideramos que es de suma importancia estudiar ambos grupos (periodistas y defensores), los cuales, además, suelen desarrollar su actividad profesional en contextos de violencia colectiva. Como se sabe, en México se han contabilizado más de 140 mil asesinatos entre diciembre de 2006 y agosto de 2015 (Mendoza \& Navarro, 2015). De 2007 a 2015, también se han reconocido oficialmente al menos 27,659 personas desaparecidas (Vicenteño, 2016); y solo durante 2014 la Comisión Interamericana de Derechos Humanos (CIDH) recibió 500 denuncias sobre violaciones graves a los derechos humanos en México (el mayor número registrado entre los países del continente americano) (Roldán, 2015).

De acuerdo con un informe de Front Line Defenders (2016), de los 156 defensores que fallecieron en todo el mundo por causas violentas en 2015, más de la mitad (87) ocurrieron en América Latina (54 en Colombia, 9 en Brasil, 8 en México, 7 en Honduras, 6 en Guatemala, 2 en Nicaragua y 1 en El Salvador). Del mismo modo, según la Comisión Interamericana de Derechos Humanos (CIDH), de 2010 a la fecha 150 periodistas fueron asesinados en América; una de cada tres de estas muertes ocurrió en México (Animal Político, 2015).

El objetivo de esta investigación de carácter descriptivo, exploratorio y transversal es identificar la prevalencia de síntomas de ETS en una muestra conjunta de periodistas mexicanos y defensores de derechos humanos que realizan su labor profesional en estrecha cercanía con víctimas de violencia. Asimismo, nos proponemos identificar si existen diferencias significativas de acuerdo con la actividad profesional (periodistas vs. defensores), género (hombres vs mujeres), horas trabajadas por semana ( $40 \mathrm{hrs}$. o menos vs más de 40 hrs.), y temáticas abordadas (incluye temática de narcotráfico vs no incluye temática de narcotráfico).

Todo lo anterior con el fin de determinar si ambos grupos (periodistas y defensores) se han visto impactados de manera secundaria por la violencia en México y reconocer si las cuatro variables mencionadas están presentes en su configuración. De manera concurrente, establecemos como propósito de esta investigación determinar la validez y confiabilidad de la Escala de Estrés Traumático Secundario de Bride et al. (2003) en esta misma muestra.

\section{Método}

\section{Participantes}

Se empleó una muestra no probabilística e intencional por invitación (Polit, 2002; Silva, 1993). En el estudio participaron 88 personas, de las cuales 29 fueron periodistas y 59 defensores de derechos humanos. Los primeros tenían la particularidad de que cubrían de manera sistemática noticias relativas a violaciones de derechos humanos, movimientos sociales y atención a víctimas de violencia (periodistas de otras fuentes fueron excluidos del estudio); mientras que los segundos participaban en distintas áreas como asesoramiento, atención y defensoría de los derechos humanos en alguna organización. Es decir, el eje común y articulador de ambos grupos era que presentaban una exposición indirecta a experiencias traumáticas de otras personas (principalmente hechos de violencia e impunidad).

El 59.1\% de la muestra estuvo constituida por mujeres y $40.9 \%$ por hombres. Una mayoría se ubicó en un rango de edad de 20 a 29 años (44.3\%). Aproximadamente la mitad de los participantes (45.5\%) solían dedicar a sus actividades profesionales más de 40 horas por semana. Por otra parte, el $83 \%$ declaró que en su labor periodística o de defensa por lo menos alguna vez habían establecido contacto con víctimas de la violencia derivada de la "guerra contra el narcotráfico". Al momento del levantamiento, el $73.9 \%$ de los participantes desempeñaba su trabajo en la ciudad de México y el resto (26.1\%) se distribuía en 8 entidades del país (Estado de México, Morelos, Oaxaca, Puebla, Veracruz, 
Chiapas, Colima y Chihuahua).

\section{Instrumentos}

Además del cuestionario sociodemográfico y laboral, se empleó la Escala de Estrés Traumático Secundario-EETS (STSS por sus siglas en inglés) desarrollada por Bride et al. (2003), la cual evalúa síntomas intrusivos, evitativos y de activación que suelen configurarse como resultado de una exposición indirecta a experiencias traumáticas.

Debido a que el instrumento no había sido validado en población mexicana, se realizó la traducción y adaptación del inglés al castellano por un grupo de expertos (todos ellos psicólogos) y, posteriormente, se determinó la validez y confiabilidad del instrumento en estos profesionistas.

La EETS consta de 17 reactivos, los cuales corresponden a la trilogía de síntomas propias del Estrés Postraumático (PTSD). No obstante, la EETS difiere de otras escalas que identifican PTSD en que justamente la mitad de sus reactivos $(2,3,6,10,12,13,14$ y 17) son ubicados específicamente como estresores derivados de una exposición indirecta al trauma lo que -como se ha señalado- constituye la esencia y particularidad del ETS.

En las instrucciones se le indica al participante que lea cada reactivo y que elija con qué frecuencia la afirmación ha sido verdadera durante los últimos siete días. El tipo de respuesta se ubica en una escala Likert que va de "1" (nunca) a " 5 " (casi siempre). De acuerdo con la versión original, un puntaje entre 28 y 37 corresponde a "sintomatología baja", 38 y 43 a "sintomatología moderada", 44 y 48 a "sintomatología alta" e igual o mayor a 49 "sintomatología severa". La puntuación total mínima del instrumento es 17 y la máxima 85.

En la EETS utilizada en esta investigación, se adaptaron ciertas palabras con el fin de focalizar el instrumento para su uso en periodistas y defensores de derechos humanos que trabajan de manera cercana con víctimas de violencia. Por ejemplo, en lugar de utilizar el sustantivo "cliente" incluido en la versión original de la escala, se optó por el de "víctima". En el texto original se menciona que la palabra "cliente" es utilizada para identificar a la persona con quien se ha establecido un vínculo en la relación de ayuda, pero que éste puede ser sustituido por otro sustantivo que mejor se ajuste o represente el trabajo del profesionista evaluado.

\section{Procedimiento}

Gracias a la intervención y apoyo de diversas organizaciones y colectivos, se convocó a sus respectivos miembros (defensores de derechos humanos y periodistas) a participar en la investigación. Se invitó directamente a 161 personas ( 74 defensores y 91 periodistas) y poco más de la mitad aceptó participar $(\mathrm{N}=88)$, configurándose una tasa de aceptación de 54\%. Quienes rechazaron participar no aceptaron contestar ni siquiera el cuestionario sociodemográfico, de tal forma que resultó imposible realizar un mínimo análisis comparativo con aquellos que sí habían decidido participar en la investigación.

A los periodistas y defensores de derechos humanos se les ofrecieron dos formas de aplicación de instrumentos: de manera tradicional (mediante el uso de papel y lápiz, y en una cita previamente determinada) o mediante el uso de una página web, la cual posibilitaba responderlos en línea. El proceso de levantamiento de datos así como la sistematización y ordenamiento de los mismos se realizó entre junio de 2014 y agosto de 2015.

\section{Consideraciones éticas}

A las organizaciones convocadas se les explicó el objetivo del estudio a través de una carta-invitación, en la que se garantizaba el resguardo de la identidad de las personas participantes. También se les proporcionaron los datos de los investigadores (teléfonos y correos electrónicos) para cualquier aclaración, duda o comentario que pudiera surgir. Los defensores y periodistas que aceptaron participar otorgaron su consentimiento informado.

\section{Análisis estadístico}

Los datos obtenidos se analizaron con el programa SPSS 19 y se emplearon diferentes estrategias estadísticas. Para obtener la validez de la escala, se utilizó el índice de adecuación de la muestra de KMO (Kaiser-Meyer-Olkin) y se realizó un análisis factorial con rotación ortogonal varimax. También se examinó la confiabilidad mediante el índice Alfa de Cronbach, y se examinaron frecuencias y porcentajes de los resultados, medias aritméticas y desviaciones estándar para identificar prevalencias. Finalmente, con el objetivo de determinar si existían diferencias entre grupos, se realizaron pruebas $t$ de Student para muestras independientes.

\section{Resultados}

\section{Análisis factorial y de confiabilidad}

Con el fin de obtener la validez de constructo de la EETS, se identificaron las frecuencias de cada pregunta y se realizó un análisis de discriminación de reactivos por el método de grupos contrastados ( $t$ de Student para muestras independientes). Se encontró que todos los ítems habían cumplido con los requerimientos necesarios, por lo que se procedió a realizar el análisis factorial. El coeficiente KMO (Kaiser-Meyer-Olkin) identificado fue de .804, y se ubicó por encima del .50 recomendado (Kaiser, 1970). 
Posteriormente, para determinar la validez del instrumento se realizó un análisis factorial con rotación ortogonal varimax. De esta forma, se obtuvieron tres factores (activación, intrusión y evitación) acordes con lo que se señala en la literatura científica sobre el tema (Bride et al., 2003). Todos los reactivos obtuvieron cargas mayores a .40 y ninguno se ubicó en más de un factor (ver Tabla 1).

Tabla 1. Factores de la Escala de Estrés Traumático Secundario

\begin{tabular}{|c|c|c|c|}
\hline Reactivos & $\begin{array}{c}\text { Factor } 1 \\
\text { Activación }\end{array}$ & $\begin{array}{l}\text { Factor } 2 \\
\text { Intrusión }\end{array}$ & $\begin{array}{l}\text { Factor } 3 \\
\text { Evitación }\end{array}$ \\
\hline 5. Me siento desanimado(a) por mi futuro. & .779 & & \\
\hline 9. Estoy menos activo(a) que de costumbre. & .778 & & \\
\hline 15. Me enojo con facilidad. & .732 & & \\
\hline 11. Tengo dificultades para concentrarme. & .650 & & \\
\hline 4. Tengo problemas para dormir. & .645 & & \\
\hline 1. Me siento emocionalmente adormecido y embotado. & .622 & & \\
\hline 8. Me siento nervioso(a). & .532 & & \\
\hline $\begin{array}{l}\text { 3. Parece como si estuviera reviviendo el trauma experimentado por } \\
\text { las víctimas. }\end{array}$ & & .861 & \\
\hline $\begin{array}{l}\text { 2. Mi corazón late rápidamente cuando pienso en las víctimas de } \\
\text { violencia. }\end{array}$ & & .808 & \\
\hline 13. Tengo sueños perturbadores sobre las víctimas. & & .529 & \\
\hline 10. Pienso en las víctimas cuando no quiero hacerlo. & & .491 & \\
\hline 16. Imagino que algo malo puede ocurrir. & & .414 & \\
\hline 14. Quiero evitar el contacto con algunas víctimas. & & & .713 \\
\hline 17. Olvido datos importantes sobre mi trabajo con víctimas. & & & 699 \\
\hline 7. Tengo poco interés en estar con otras personas. & & & 618 \\
\hline $\begin{array}{l}\text { 12. Evito el contacto con gente, lugares o cosas que me recuerdan a } \\
\text { las víctimas. }\end{array}$ & & & .517 \\
\hline 6. Los recuerdos sobre las víctimas me alteran. & & & .443 \\
\hline Valor Eigen & 3.87 & 2.56 & 2.53 \\
\hline Porcentaje de varianza explicada & 22.79 & 15.10 & 14.88 \\
\hline
\end{tabular}

Fuente: Elaboración propia.

Por otra parte, la media del puntaje total del instrumento fue 39.57 (DE = 10.31), lo cual en promedio ubicó a los participantes con niveles moderados de ETS. En cuanto a los síntomas desagregados, se identificaron las siguientes medias: intrusión 10.93 (DE = 3.50), evitación $10.01(\mathrm{DE}=3.30)$ y activación $18.63(\mathrm{DE}=$ 5.66).

Se encontró que utilizando un punto de corte de 44 (es decir, con puntajes que ubicaron a los participantes con sintomatología "alta" o "severa"), la prevalencia global de síntomas de ETS en esta muestra fue del $36.4 \%$. En cuanto a los indicadores desagregados por actividad laboral, se identificó que el $44.8 \%$ de los periodistas presentaba síntomas de ETS, mientras que estos síntomas afectaron a los defensores de derechos
El Alfa de Cronbach total del instrumento fue .87, y la varianza total explicada $52.78 \%$. El coeficiente de confiabilidad del primer factor (activación) fue .84, del segundo .72 (intrusión) y del tercero .69 (evitación). Salvo este último, todos se ubicaron por encima del .70 recomendado (Nunnally \& Berstein, 1994). 
Tabla 2. Diferencias por género, actividad profesional, horas trabajadas por semana y temáticas abordadas en cuanto a los puntajes totales de la EETS

\begin{tabular}{|c|c|c|c|c|c|}
\hline M & DE & M & DE & $t$ & p \\
\hline \multicolumn{2}{|c|}{ Mujeres } & \multicolumn{2}{|c|}{ Hombres } & & \\
\hline 41.35 & 9.70 & 37 & 10.74 & -1.97 & 0.05 \\
\hline \multicolumn{2}{|c|}{ Periodistas } & \multicolumn{2}{|c|}{ Defensores(as) } & & \\
\hline 40.90 & 10.65 & 38.92 & 10.16 & 0.84 & 0.40 \\
\hline \multicolumn{2}{|c|}{40 horas o menos } & \multicolumn{2}{|c|}{ Más de 40 horas } & & \\
\hline 37.54 & 10.20 & 42 & 10.03 & -2.05 & 0.04 \\
\hline \multicolumn{2}{|c|}{ ITN } & \multicolumn{2}{|c|}{ NITN } & & \\
\hline 40.30 & 10.64 & 36 & 7.87 & -1.48 & 0.14 \\
\hline
\end{tabular}

Fuente: Elaboración propia.

$\mathrm{M}=$ Media aritmética.

$\mathrm{DE}=$ Desviación estándar.

ITN= Incluye temáticas de narcotráfico.

NITN $=$ No incluye temáticas de narcotráfico.

hombres, así como también aquellos que trabajan más de 40 horas por semana.

Por otra parte, en la Tabla 3 se presentan los resultados obtenidos al realizar pruebas t de Student por grupo de síntomas (activación, intrusión y evitación) de acuerdo con el género, actividad profesional, horas trabajadas a la semana y temáticas abordadas.

Como puede observarse, al considerar el género, solamente se encontraron diferencias en los síntomas intrusivos, siendo las mujeres quienes obtuvieron una media significativamente más alta que los hombres. De la misma forma, en cuanto a la presencia de síntomas evitativos, se identificaron puntajes significativamente más elevados en aquellas personas cuya carga horaria superaba las 40 horas semanales. De igual mane$\mathrm{ra}$, los profesionales que desarrollaban en su trabajo temáticas de narcotráfico se ubicaron con puntajes de evitación significativamente más altos que aquellos que no lo hacían. Por último, al realizar comparaciones en el resto de los grupos, no se encontraron diferencias estadísticamente significativas en ninguna otra área sintomática.

\section{Discusión}

De acuerdo con los objetivos planteados en esta investigación, se encontró que la Escala de Estrés Traumático Secundario (EETS) de Bride et al. (2003) cumplió con los estándares necesarios de validez y confiabilidad en periodistas y defensores mexicanos. Este hecho resulta de singular importancia, ya que contar con un instrumento estandarizado para dichos profesionistas posibilita que en futuras investigaciones se puedan evaluar síntomas de ETS en ambos grupos, y relacionarlos con otras posibles variables de interés clínico y profesional como la ansiedad, la depresión, el burnout, la resiliencia o el crecimiento postraumático.

Por otra parte, la prevalencia global de síntomas de ETS identificada en este estudio fue del $36.4 \%$. Es decir, se encontró que, en promedio, uno de cada tres participantes presentó sintomatología "alta" o "severa". Estos indicadores demuestran el considerable impacto que pueden producir las exposiciones traumáticas secundarias en el bienestar psicológico de periodistas y defensores de derechos humanos. Sin embargo, no se encontraron diferencias estadísticamente significativas entre ambos grupos, lo cual indica que el impacto tiende a ser homogéneo pese a que los periodistas presentaron un porcentaje más alto de síntomas de ETS que los defensores. En otros términos, podría afirmarse que la experiencia indirecta de tipo violento es de tal magnitud que su impacto repercute de manera similar en ambos grupos, independientemente de las labores y actividades específicas que realizan con las víctimas de violencia.

Aunque resulta considerablemente elevada la prevalencia de síntomas en ambos grupos, ésta se ubica ligeramente por debajo de la identificada en psicólogos clínicos (37.8\%) (Guerra et al., 2009), es equivalente a la de trabajadores de servicios médicos (36.5\%) (Montero, 2014), y está por encima de la encontrada en enfermeras (35\%) (Beck \& Gable, 2012). En cualquier caso, son cifras que muestran un impacto considerable.

No obstante, consideramos que también puede realizarse una interpretación invertida de las prevalencias. Esto es, podemos admitir que -pese al considerable impacto emocional experimentado durante su 
Tabla 3. Diferencias por género, actividad profesional, horas trabajadas por semana y temáticas abordadas, en cuanto a la presencia de síntomas de activación, intrusión y evitación

\begin{tabular}{lllllll}
\hline M Total & Grupo & M Grupo 1 & $D E$ & M Grupo 2 & $D E$ & $t$ \\
\hline
\end{tabular}

Fuente: Elaboración propia

$\mathrm{M}=$ Media aritmética.

$\mathrm{DE}=$ Desviación estándar.

ITN= Incluye temáticas de narcotráfico.

NITN $=$ No incluye temáticas de narcotráfico

trabajo diario- un grupo mayoritario de periodistas y defensores de derechos humanos registró niveles bajos de sintomatología. Ciertamente, el dato complementario de que el $63.6 \%$ de los periodistas y defensores presentaran sintomatología "leve" o "moderada" nos permite inferir la presencia de ciertos factores resilientes en ellos. Valdría la pena, en consecuencia, investigar a profundidad algunas de estas características en futuros estudios. Podrían estar interviniendo, por ejemplo, variables sociodemográficas específicas (como la edad, zona de residencia, etc.), ciertas activ-

idades autoprotectoras (como el ejercicio físico o la realización de actividades culturales o lúdicas ajenas a su actividad profesional) o la presencia de algún tipo de proceso psicoterapéutico entre muchas otras razones.

Respecto a las diferencias por género en cuanto a los niveles de impacto, consideramos que es importante destacar dos situaciones. En primer término, el hecho de que las mujeres han estado jugando en México un papel cada vez más relevante en la denuncia de la vio- 
lencia, ya sea a través del periodismo o bien a partir de la defensa de los derechos humanos. Este protagonismo se manifiesta en los múltiples movimientos sociales encabezados por mujeres, quienes -inclusive- suelen provenir de una transformación desde la victimización hacia el activismo (López, 2014; Medina, 2012). En segundo lugar y unido a lo anterior, se observa que las mujeres van solidarizándose con las víctimas generadas por la violencia, convirtiéndose en una suerte de acompañantes y guías con los subsiguientes impactos generados por la exposición a nuevos traumas. Burgos et al. (2014) han señalado esta idea en distintos foros, reivindicando particularmente la importancia del autocuidado en mujeres defensoras como herramienta esencial para el fortalecimiento de los movimientos sociales.

Las situaciones arriba señaladas podrían explicar en conjunto la presencia de puntajes altos en mujeres (principalmente de síntomas intrusivos). En términos coloquiales, podría afirmarse que a las mujeres de este estudio -a diferencia de los varones- les ha resultado más difícil "quitarse de la cabeza" y "marcar distancia" respecto a las experiencias de dolor de las víctimas con quienes establecieron contacto. En efecto, numerosas son las autoras que han analizado las particularidades de la subjetividad femenina, ya sea en procesos de elaboración traumáticos o en manifestaciones de bondad y empatía (Garriga, 2014; Gilligan, 2013; Magaña, Silva-Nadales \& Rovira, 2010). No obstante, consideramos que resulta necesario desarrollar más estudios de corte cualitativo en mujeres periodistas y defensoras de derechos humanos mexicanas con el fin de analizar en profundidad las razones de estas diferencias intrusivas específicas.

Por su parte, la falta de diferenciación significativa en puntajes globales respecto a la temática del narcotráfico remite sobre todo al carácter psicosocial de este tipo de traumas. Es decir, permite dimensionar el impacto psicológico que está generando la violencia en México -y no solo la relacionada con hechos de narcotráfico-, así como la crisis de derechos humanos que se vive en el territorio nacional.

Sin embargo, llama particularmente la atención el mayor desarrollo de síntomas evitativos en quienes trabajan temáticas de narcotráfico. Dichos síntomas, en efecto, dan cuenta del impacto traumático, pero, paradójicamente, también constituyen factores protectores del psiquismo; es decir, podrían representar un intento de cesar la exposición a las historias y/o situaciones derivadas de su trabajo diario que, de algún modo, están afectándolos psicológicamente. Con respecto a las respuestas de evitación propias del Estrés Postraumático (PTSD), van der Kolk (2007) señala lo siguiente:

Puede ser posible que sean precisamente estos elementos excluidos, que son las sensaciones a las que el paciente evita enfrentarse activamente y revivir en la relación terapéutica, los que sean más proclives a reaparecer en forma de escenas retrospectivas (destellos rememorativos), pesadillas y reactuaciones conductuales. $(p .11)$

No obstante, las conductas de evitación también pueden ser el resultado de la necesidad objetiva de tomar medidas de precaución ante el peligro que representa trabajar temáticas relacionadas con el narcotráfico y el crimen organizado. No debe soslayarse que, en efecto, el número de periodistas y defensores amenazados y agredidos ha ido en aumento durante los últimos años como se ha señalado en la introducción del presente texto.

Por otra parte, en este estudio se confirmó que uno de los factores que contribuyen al desarrollo de ETS es la sobrecarga horaria. Se identificó que los participantes (tanto periodistas como defensores) que laboraban más de 40 horas a la semana presentaron más síntomas, lo cual constituye -independientemente de las tareas específicas que realizan- un factor de riesgo para el desarrollo de ETS. Si a ello agregamos el impacto en el psiquismo de la exposición sistemática a las historias de dolor de las víctimas, el riesgo aumenta considerablemente.

Cabe señalar que el tratamiento de los datos no arrojó diferencias significativas en cuanto a síntomas de activación. No obstante, la media para este criterio específico de ETS fue de 18.63, lo cual indica una presencia importante de sintomatología relativa a las emociones: desánimo, irritabilidad, trastornos del sueño, nerviosismo y embotamiento. Si bien lo anterior pone el acento en las condiciones laborales, no hay que olvidar el riesgo para la propia integridad personal que representa el contexto de violencia en el que se desenvuelven periodistas y defensores, tal y como ha quedado expuesto en la introducción.

Por último, a partir de los datos analizados en este estudio, surgen nuevas interrogantes que precisan ser investigadas bajo modalidades mixtas: ¿qué medidas de autoprotección psicológica deben desarrollar periodistas y defensores al realizar su trabajo diario?, ¿cómo se puede brindar atención a las víctimas directas e indirectas de la violencia en México sin que ello represente riesgos para la salud mental y para los derechos humanos de los trabajadores que prestan dichos servicios?, ¿qué medidas estarían bajo la responsabilidad del Estado y otros actores privados para garantizar el apoyo a las víctimas en consonancia con condiciones laborales dignas para los trabajadores?

Además de la contribución que representa la validación de la EETS y las prevalencias aquí identificadas, consideramos que este estudio coloca en la mesa de discusión la problemática del impacto secundario en México, cuyos efectos han sido subestimados 
por la hegemonía que se ha concedido a las exposiciones traumáticas de carácter primario. Los resultados globales de esta investigación revelan, en suma, el considerable desgaste psicoemocional que generan las exposiciones secundarias en la subjetividad de quienes documentan y establecen vínculos sistemáticos con personas traumatizadas por la creciente ola de violencia que se ha vivido en México durante las últimas dos décadas.

\section{Referencias}

Adam, R., Boscarino, J. \& Figley, C. (2006). Compassion Fatigue and Psychological Distress Among Social Workers: A Validation Study. The American Journal of Orthopsychiatry, 76(1), 103-108. doi: 10.1037/0002-9432.76.1.103

Adams, R., Figley, C. \& Boscarino, J. (2008). The compassion fatigue scale: its use with social workers following urban disaster. Research on Social Work Practice, 18(3), 238-250. doi: 10.1177/1049731507310190

Animal Político. (2 de noviembre de 2015). Uno de cada tres asesinatos de periodistas en América ocurrió en México: CIDH. Animal Político. Recuperado de http://www.animalpolitico.com/2015/11/ uno-de-cada-tres-asesinatos-de-periodistas-en-america-ocurrio-en-mexico-cidh/

Beck, C. \& Gable, R. (2012). A Mixed Methods Study of Secondary Traumatic Stress in Labor and Delivery Nurses. Journal of Obstetric, Gynecologic, \& Neonatal Nursing, 41(6), 747-760. doi: 10.1111/j.1552-6909.2012.01386.x.

Betta, R., Morales, G., Rodríguez, K. \& Guerra, C. (2007). La frecuencia de emisión de conductas de autocuidado y su relación con los niveles de estrés traumático secundario y de depresión en psicólogos clínicos. Pensamiento Psicológico, 3(9), 9-19. Recuperado de http://www.redalyc. org/pdf/801/80103902.pdf

Brady, J., Guy, J., Poelstra, P. \& Brokaw, B. (1999). Vicarious traumatization, spirituality, and the treatment of sexual abuse survivors: A national survey of women psychotherapists. Professional Psychology: Research and Practice, 30, 386393. doi: 10.1037/0735-7028.30.4.386

Briere, J. \& Scott, C. (2006). Principles of trauma therapy: A guide to symptoms, evaluation, and treatment. Thousand Oaks, CA: Sage.

Bride, B., Robinson, M., Yegidis M. \& Figley, C. (2003). Development and validation of the secondary traumatic stress scale. Research on Social Work Practice, 14, 27-35. doi: $10.1177 / 1049731503254106$
Burgos, A., Hernández, A., Herraez, A., Ramos, G., Burgos, L., Zelaya, L., ... \& Arauz, Z. (2014). Travesías para pensar y actuar. Experiencias de autocuidado de defensoras de derechos humanos en Mesoamérica. Oaxaca: I-M Defensoras.

Chrestman, K. (1999). Secondary exposure to trauma and self reported distress among therapists. En B. Stamm (Ed.), Secondary traumatic stress: Self-care issues for clinicians, researchers, and educators (2nd ed., pp. 29-36). Lutherville, MD: Sidran.

Courtois, C. (1988). Healing the incest wound: Adult survivors in therapy. New York: Norton.

Cunningham, M. (2003). Impact of trauma work on social work clinicians: Empirical findings. Social Work, 48, 451-459. doi: 10.1093/sw/48.4.451

Dunkley, J. \& Whelan, T. (2006). Vicarious traumatization: Current status and future directions. British Journal of Guidance and Counseling, 34(1), 107-116. doi:10.1080/03069880500483166

Fernández-Pinto, I., López-Pérez, B. \& Márquez, M. (2008). Empatía: Medidas, teorías y aplicaciones en revisión. Anales de Psicología, 24(2), 284-298. Recuperado de http://www.um.es/analesps/v24/v24_2/12-24_2.pdf

Figley, C. (Ed.). (1995). Compassion fatigue. Coping with secondary traumatic stress disorder in those who treat the traumatized. New York: Brunner/Mazel.

Figley, C. (1999). Compassion fatigue: Toward a new understanding of the cost of caring. En B. Stamm (Ed.). Secondary traumatic stress: Self-care issues for clinicians, researchers, and educators (2nd ed., pp. 3-28). Lutherville, MD: Sidran.

Flores-Morales, R. \& Reidl, L. (2014). Traumatización secundaria en periodistas que cubrieron la caravana por la paz. En S. Rivera, P. Wolfgang, L. Cruz \& G. Villanueva (Eds.), Aportaciones Actuales de la Psicología Social Volumen 2 (pp. 814-821). México D.F.: Asociación Mexicana de Psicología Social.

Follette, V., Polusny, M. \& Milbeck, K. (1994). Mental health and law enforcement professionals: Trauma history, psychological symptoms, and impact of providing services to child sexual abuse survivors. Professional Psychology: Research and Practice, 25(3), 275-282. doi: 10.1037/0735-7028.25.3.275

Front Line Defenders. (2016). Annual Report 2016. Recuperado de https://frontlinedefenders.org/ sites/default/files/report.pdf

Gardell, D. \& Harris, D. (2003). Childhood abuse histo- 
ry, secondary traumatic stress, and child welfare workers. Child Welfare, 82(1), 5-26.

Garriga, C. (2014). La bondad y la ética del cuidado en la subjetividad femenina. Implicaciones del DSM-V para la sexualidad de las mujeres. Aperturas psicoanalíticas, 46, 382-396. Recuperado de http://www.aperturas.org/articulos. php?id $=0000843$

Gilligan, C. (2013). La ética del cuidado. Barcelona: Fundació Víctor Grífols i Lucas.

Guerra, C., Fuenzalida, A. \& Hernández, C. (2009). Efectos de una Intervención Cognitivo-Conductual en el Aumento de Conductas de Autocuidado y Disminución del Estrés Traumático Secundario en Psicólogos Clínicos. Terapia Psicológica, 27(1), 73-81. doi: 10.4067/S071848082009000100007

Guerra, C. \& Pereda, N. (2015). Estrés traumático secundario en psicólogos que atienden a niños y niñas víctimas de malos tratos y abuso sexual: un estudio exploratorio. Anuario de Psicología/The UB Journal of Psychology, 45(2), 177-188. Recuperado de http://revistes.ub.edu/ index.php/Anuario-psicologia/article/viewFile/14265/17522

Hatfield, E., Cacciopo, J., \& Rapson, R., (1994). Emotional Contagion. Cambridge: Cambridge University Press.

Hensel, J., Ruiz, C., Finney, C. \& Dewa, C. (2015). Meta-Analysis of Risk Factors for Secondary Traumatic Stress in Therapeutic Work With Trauma Victims. Journal of Traumatic Stress Studies, 28(2), 83-91. doi: 10.1002/jts. 21998.

Herman, J. (1992). Trauma and recovery: The aftermath of violence from domestic abuse to political terror. New York: Basic Books.

Horowitz, (1993). Stress-response syndromes: A review of posttraumatic stress and adjustment disorders. En J. Wilson \& B. Raphael (Eds.), International handbook of traumatic stress syndromes. New York: Plenum.

Joinson, C. (1992). Coping with compassion fatigue. Nursing, 22(4), 116-120 .

Kaiser, H. (1970). A second generation little jiffy. Psychometrika, 35, 401-416. doi: 10.1007/BF02291817

Lerias, D. \& Byrne, M. (2003). Vicarious traumatization: Symptoms and predictors. Stress and Health, 19(3), 129-138. doi: 10.1002/smi.969

López, M. (2014). Abriendo caminos de libertad e igualdad. México: Iniciativa Mesoamericana de mujeres defensoras de derechos humanos.
Magaña, I., Silva-Nadales, S. \& Rovira, R. (2010). Catástrofe, subjetividad femenina y reconstrucción: Aportes y desafíos desde un enfoque de género para la intervención psicosocial en comunidades afectadas por el terremoto. Terapia psicológica, 28(2), 169-177. doi: 10.4067/S071848082010000200005

McCann, I. \& Pearlman, L. (1990). Vicarious traumatization: A framework for understanding the psychological effects of working with victims. Journal of Traumatic Stress, 3(1), 131-149. doi: 10.1007/BF00975140

Meda, R., Moreno, B., Palomera, A., Arias, E. \& Vargas, R. (2012). La Evaluación del Estrés Traumático Secundario. Estudio Comparado en Bomberos y Paramédicos de los Servicios de Emergencia de Guadalajara, México. Terapia Psicológica, 30(2), 31-41. doi: $10.4067 /$ S0718-48082012000200003

Meda, R., Moreno, B., Rodríguez, A., Arias, E. \& Palomera, A. (2011). Validación mexicana de la Escala de Estrés Traumático Secundario. Psicología y Salud, 21(1), 5-15. doi: 10.4067/S071848082012000200003

Medina, A. (2012). Defensoras de derechos humanos en México. Diagnóstico 2010-2011 sobre las condiciones y riesgos que enfrentan en el ejercicio de su trabajo. México D.F.: JASS.

Mendoza, E. \& Navarro, A. (2015, agosto 30). 32 meses: 57,410 asesinatos. Proceso, 17-19 .

Meyers, T. \& Cornille, T. (2002). The trauma of working with traumatized children. En C. Figley (Ed.), Treating compassion fatigue (pp. 39-55). New York: Brunner Routledge.

Montero, F. (2014). Estudio de factores psicológi$\cos$ y conductas de autocuidado asociados a procesos de estrés traumático secundario en profesionales de la salud mental. (Tesis de maestría inédita). Universidad de la República, Montevideo, Uruguay.

Moreno, B., Morante, M., Garrosa, E. \& Rodríguez, E. (2004a). El estrés traumático secundario. Evaluación, prevención e intervención. Terapia Psicológica, 22(1), 69-76. Recuperado de http:// www.redalyc.org/pdf/785/78522108.pdf

Moreno, B., Morante, M., Garrosa, E. \& Rodríguez, E. (2004b). Estrés traumático secundario: el coste de cuidar el trauma. Psicología conductual, 12(2), 215-231. Recuperado de https://www. uam.es/gruposinv/esalud/Articulos/Salud\%20 Laboral/2004el-coste-cuidar-el-traumapsconductual.pdf

Moreno, B., Morante, M., Rodríguez, R. \& Rodríguez, 
A. (2008). Resistencia y vulnerabilidad ante el trauma: el efecto moderador de las variables de personalidad. Psicothema, 20(1), 124130. Recuperado de http://www.redalyc.org/ pdf/727/72720117.pdf

Nunnally, J. \& Berstein, I. (1994). Psychometric theory (3rd ed.). New York: McGraw-Hill.

Pearlman, L. \& Saakvitne, K. (1995). Treating therapists with vicarious traumatization and secondary traumatic stress disorders. En C. Figley (Ed.), Compassion fatigue: Coping with secondary traumatic stress disorder in those who treat the traumatized (pp. 150-177). Bristol, P. A: Brunner/ Mazel.

Pines, A. \& Aronson, E. (1988). Career burnout: causes and cures. New York: Free Press.

Polit, H. (2002). Investigación científica en ciencias de la salud. México: McGraw-Hill Interamericana.

Roldán, N. (15 de mayo de 2015). México, el país con más denuncias de violaciones de derechos ante la CIDH. Animal Político. Recuperado de http:// www.animalpolitico.com/2015/05/mexico-elpais-con-mas-denuncias-de-violaciones-dederechos-ante-la-cidh/

Rossi, A., Cetrano, G., Pertile, R., Rabbi, L., Donisi, V., Grigoletti, L., ... \& Amaddeo, F. (2012). Burnout, compassion fatigue, and compassion satisfaction among staff in community-based mental health services. Psychiatry Research, 200(2-3), 933-938. doi: 10.1016/j.psychres.2012.07.029

Silva, L. (1993). Muestreo para la investigación en ciencias de la salud. Madrid: Díaz de Santos.

Smither, S. (1977). A reconsideration of the developmental study of empathy. Human development, 20, 253-276. doi: 10.1159/000271561

Solomon, A., Waysman, M., Belkin, R., Levy, G., Mikulincer, M. \& Enoch, D. (1992). Marital relations and combat stress reaction: The wive's perspective. Journal of Marriage and Family Therapy, 54(2), 316-326. doi: 10.2307/353063

van der Kolk, B. (2007). Más allá de la cura por el diálogo: experiencia somática, improntas subcorticales y tratamiento del trauma. Revista de toxicomanías, 51, 3-21. Recuperado de http://www. cat-barcelona.com/uploads/rets/Ret51_1.pdf

van der Kolk, B., McFarlane, A. \& Weisaeth, L. (Eds.). (2007). Traumatic stress. The effects of overwhelming experience on mind, body and society. New York: The Guilford Press.

Vicenteño, D. (11 de febrero de 2016). Hay 27 mil 659 desaparecidos; reporte oficial del gobierno.
Excélsior. Recuperado de http://www.excelsior. com.mx/nacional/2016/02/11/1074536

Wilson, J. \& Raphael, B. (Eds.). (1993). International handbook of traumatic stress syndromes. New York: Plenum. 